\title{
LITERATURBERICHT
}

\author{
Simone Wisotzki
}

\section{Notfalls mit Gewalt? Globale Gerechtigkeit und die Rechtfertigung militärischer Intervention}

Der Literaturüberblick befasst sich mit neueren Debatten der internationalen Ethik und globalen Gerechtigkeit zu Fragen militärischer Intervention. Sowohl in der Internationalen Politischen Theorie (IPT) als auch in den Theorien der Internationalen Beziehungen (IB) finden sich Ansätze, die Fragen der Verantwortlichkeit von Staaten jenseits eigener staatlicher Grenzen und der Reichweite der moralischen Verpflichtung zur Solidarität mit notleidenden Menschen kontrovers diskutieren. In der Debatte über den moralisch gerechtfertigten Einsatz von Gewalt wird darüber gestritten, inwieweit der Schutz individueller Menschenrechte höher zu bewerten ist als fundamentale Normen der Souveränität. Während sich aus kosmopolitischer Perspektive gerechte Gründe für den Gewalteinsatz ergeben, monieren kritische Ansätze die liberale Hierarchisierung globaler Gerechtigkeit. Diskursethische Verfahren zeigen Auswege auf, sind aber ebenfalls mit Schwierigkeiten konfrontiert. Für die Friedens- und Konfliktforschung sind diese Diskussionen von Interesse, weil Gerechtigkeitsargumente von politischer Seite zur Überzeugung der öffentlichen Meinung herangezogen werden und auf diese Weise Rechtfertigungsstrategien für Gewalthandeln eine beständige Erweiterung erfahren.

Schlagworte: Internationale Ethik, Globale Gerechtigkeit, Theorie des »Gerechten Krieges«, Humanitäre Intervention

\section{Einleitung}

Die Debatten um eine globale Ethik für internationale Beziehungen und Fragen der globalen Gerechtigkeit haben in jüngster Zeit an Bedeutung gewonnen (Shapcott 2010; Hutchings 2010; G. Brock 2009; Frost 2009; Appiah 2006). Die Frage nach der Verantwortlichkeit jenseits der eigenen staatlichen Grenzen und die moralische Verpflichtung zur Solidarität mit notleidenden Menschen stehen dabei oftmals im Mittelpunkt der Diskussion. Der Literaturüberblick will sich einem Ausschnitt die- 
ser sehr viel breiteren Debatte zu internationaler Ethik und globaler Gerechtigkeit widmen. Die Frage nach der Verantwortung jenseits der eigenen staatlichen Grenzen umfasst auch das Themenfeld der Migration und das Recht auf Freizügigkeit (Shapcott 2010: 151; G. Brock 2009: 190-212). Aus der Perspektive der globalen Verteilungsgerechtigkeit geht es etwa um die Frage der Beseitigung von Armut und globalen Disparitäten sowie grundlegend um eine gerechte globale (Weltwirtschafts-)Ordnung. ${ }^{1}$

Hier soll jedoch der Frage nachgegangen werden, inwieweit sich aus einer Perspektive globaler Gerechtigkeit eine Verantwortung von Staaten oder der internationalen Staatengemeinschaft ableiten lässt, die einen Gewalteinsatz, beispielsweise im Fall schwerer Menschenrechtsverletzungen, und somit eine militärische Intervention moralisch rechtfertigen.

Die Debatte um eine internationale Ethik des Einsatzes von Gewalt wird dabei vom Spannungsfeld zwischen dem Schutz individueller Menschenrechte und der Wahrung staatlicher Souveränität bestimmt. Dabei wird argumentiert, dass die soziale Realität längst kosmopolitisch geworden sei und der Schutz individueller Menschenrechte höher zu bewerten sei als fundamentale Normen der Souveränität, Nichteinmischung und Nichtintervention. Der Streit zwischen Vertretern verschiedener Denkschulen dreht sich um die Frage, ob staatliche Souveränität angesichts globaler Interdependenzen einen Anachronismus darstellt und militärische Interventionen gerade bei schweren Menschenrechtsverletzungen als moralische Verpflichtung der internationalen Staatengemeinschaft angesehen werden muss, die selbst dann geboten ist, wenn die rechtliche Instanz des UN-Sicherheitsrates darüber keine Einigung erzielt. Moralische Rechtfertigungsstrategien, wie der Schutz von Menschenrechten, wurden unter anderem nach dem NATO-Einsatz im Kosovo im Hinblick auf die Legitimität und Legalität der Militärintervention in der Literatur diskutiert (Hehir 2010: 83-104; Falk 2005: 36-41). Selbst der Irak-Krieg 2003 wurde vom damaligen britischen Premierminister Tony Blair unter anderem mit Verweis auf die schwereren Menschenrechtsverletzungen des autoritären Unrechtsregimes Saddam Husseins moralisch gerechtfertigt. Auch die Idee der sogenannten »Schutzverantwortung « (responsibility to protect - R2P) gründet auf dem Nexus zwischen

1 Für wertvolle Anregungen und Kritik bedanke ich mich bei Carolin Anthes, Tanja Brühl, Cora Ditzel, Nicholas Galbenis, Gregor Hofmann, Harald Müller, Peter Schlotter sowie den anonymen GutachterInnen. Die Auswahl aus der umfangreichen Literatur zu diesem Thema kann nur selektiv und damit subjektiv aus der Perspektive der Autorin erfolgen. Mögliche Versäumnisse fallen allein in die Verantwortung der Autorin. Des Weiteren verzichtet der vorliegende Artikel auf eine geschlechtersensible Sprache; vielmehr ist die Geschlechterperspektive als Teil des Literaturüberblicks systematisch berücksichtigt worden. 
der Souveränität von Staaten und deren Verantwortung für ihre Bürger (MacFarlane et al. 2004). Neben dieser »inneren Verantwortung « kommt in der Gerechtigkeitsliteratur immer wieder die Frage nach der »kollektiven « oder »äußeren Verantwortung « der internationalen Staatengemeinschaft auf, die besonders dann relevant wird, wenn Staaten diesen Anforderungen nicht nachkommen und systematisch mit Gewalt gegen die eigene Bevölkerung vorgehen (Stahn 2007: 112; Welsh 2010: 273; Bellamy 2009; Evans 2009). ${ }^{2}$ Solche Bemühungen um eine stärkere Konstitutionalisierung von Menschenrechten im Hinblick auf den Einsatz von Gewalt generieren Ressentiments in den Reihen der Staaten des globalen Südens. Immer wieder werden dabei westliche Doppelstandards und die Selektivität in der bisherigen Interventionspraxis angeprangert (Ayoob 2004: 110; Bellamy 2005). Gleichzeitig begreifen zahlreiche dieser Staaten Souveränität und Nichteinmischung als Institutionen freier Menschen und Errungenschaften des postkolonialen Zeitalters und somit keineswegs als Anachronismus, sondern vielmehr als Gerechtigkeitsanspruch (Bajpai 2003: 260).

Solche Unterschiede in den Gerechtigkeitsauffassungen zwischen Staaten bestimmen nicht nur die politischen Debatten in den internationalen Beziehungen, sondern werden auch in der Literatur zur internationalen Ethik breit reflektiert. Der folgende Literaturüberblick will sich den Darlegungen moralischer Rechtfertigungen und differenter Gerechtigkeitsvorstellungen im Hinblick auf den Einsatz von Gewalt primär aus einer theoretischen Perspektive widmen. Im Mittelpunkt der Betrachtungen stehen darum Debatten im Rahmen der Internationalen Politischen Theorie (IPT) und der internationalen Ethik sowie der Theorien Internationaler Beziehungen (IB). Die Auswahl der einzelnen Denkschulen begründet sich dabei primär durch den Themenschwerpunkt - die Benennung gerechter Gründe für die Rechtfertigung militärischer Gewalt. Die Debatten zur internationalen Ethik werden auch unter dem Stichwort $»$ normative IR theory $\ll^{3}$ zusammengefasst, weil sie auf der Grundlage eigener Werturteile Sollensansprüche für die internationale Politik entwickeln und Handlungsanleitungen geben. ${ }^{4}$ Als zentrales Element findet sich in der Literatur auch eine Wiederbelebung der Kategorien des »Gerechten Krieges«.

2 Stahn (2007: 112) rekapituliert den Prozess der Normgenese der R2P. Er argumentiert, dass mit der Schutzverantwortung der Nexus zwischen staatlicher Souveränität und innerer wie äußerer Verantwortung von Staaten normativ gestärkt worden sei.

3 »IR« steht für »International Relations « und ist die englische Bezeichnung für die politikwissenschaftliche Teildisziplin »Internationale Beziehungen« (IB).

4 Vertreter normativer Theorien wollen sich damit von solchen IB-Theorien abgrenzen, die einen Erklärensanspruch verfolgen (Erskine 2008: 9). Brown (2002: 3; 1992) wendet sich gegen die Differenzierung von »normativ« und "positivistisch« und merkt kritisch an, dass die Formulierung 
Für die Friedens- und Konfliktforschung sind solche Debatten in mehrfacher Sicht spannend und somit fürden vorliegenden Literaturüberblick strukturgebend. ${ }^{5}$ So zeigt sich in denüberwiegend kosmopolitisch geprägten Diskursen derIPT, aberauch in den Ansätzen der IB, dass Gerechtigkeitsargumente zur Begründung einer Vielzahl von Interventionen herangezogen werden und hierüber die Rechtfertigungsgründe fürGewalthandeln eine beständige Erweiterung erfahren. Gefragt wird nicht mehr, ob ein Krieg legal ist, sondern ob er sich moralisch rechtfertigen lässt. Kapitel 2 bietet zunächst einen kursorischen Überblick über die verschiedenen Argumentationsstränge innerhalb des Kosmopolitismus und die Differenzen zum Kommunitarismus im Hinblick auf die Frage nach der Verantwortung von Staaten jenseits der eigenen Grenzen. In Kapitel 3 wird dann aus der Perspektive der IPT diese Verantwortung von Staaten im Hinblick auf einen möglichen Gewalteinsatz ausbuchstabiert und der Argumentationsgang zu den gerechten Gründen militärischer Gewalt entfaltet. Gleiches erfolgt dann in Kapitel 4 aus der Perspektive ausgewählter Theorien der IB. Kapitel 3 und 4 sollen auch verdeutlichen, dass sowohl in der IPT als auch in den Theorien der IB auf der Grundlage einer liberalen Logik der Menschenrechte und eines ethischen Individualismus plurale Rechtfertigungsstrategien für Gewalthandeln entworfen werden, die nicht nur auf theoretischer Ebene zur Entgrenzung legitimer Gründe für militärische Interventionen führen, sondern sich auch dazu eignen, von politischer Seite als Rechtfertigungsstrategie und Überzeugungsargumentation gegenüber der Öffentlichkeit herangezogen zu werden. Auf diese Weise lässt sich selbst der militärisch erzwungene demokratische Regimewechsel auf der Grundlage von Argumentationsfiguren aus der internationalen Ethik rechtfertigen. Kritische Ansätze betonen die Idiosynkrasien einer solchen moralisch gerechtfertigten Weltordnungspolitik und verweisen auf den distinkten historisch-kulturellen, westlichen Kontext, in dem die Mehrzahl der theoretischen Überlegungen entstanden ist, wie in Kapitel 5 herausgearbeitet wird. Der Literaturüberblick endet mit einem Plädoyer für eine stärker empirisch-analytisch ausgerichtete Gerechtigkeitsforschung, die sich darauf konzentriert, die realpolitisch vorhandenen Differenzen und Friktionen zwischen Staaten im Hinblick auf die jeweiligen Gerechtigkeitsvorstellungen zu eruieren, und Reichweite und Grenzen kollektiver Verantwortung mit Blick auf den Einsatz militärischer Gewalt zu ermitteln.

moralischer Sollensansprüche nicht in jedem Fall ein konstitutives Element normativer Ansätze darstellt. Andere bezeichnen mit der normativen Theorie internationaler Beziehungen die Debatte zwischen Kosmopolitismus und Kommunitarismus (Cochran 1999: XV).

5 Auch die Arbeitsgemeinschaft fürFriedens-und Konfliktforschung(AFK)hatsich in ihren Kolloquien wiederholt mit dem Thema beschäftigt. Siehe dazu exemplarisch Imbusch(2007) sowie Jaberg/Schlotter (2005). 


\section{Globale Gerechtigkeit und die Verantwortung jenseits eigener Grenzen: Die Debatte zwischen Kosmopolitismus und Kommunitarismus}

Die ethische Perspektive in den IB beschäftigt sich allgemein mit moralwissenschaftlichen Fragestellungen, konkret mit den Prinzipien menschlicher Pflichten jenseits nationalstaatlicher Grenzen, auch gegenüber »Fremden« (Shapcott 2010: 4). Moral wird dabei verstanden als eine Form praktischen, intersubjektiven Wissens, welches richtiges Handeln in Entscheidungssituationen anleitet (Habermas 1999 a: 273; Hasenclever 2000: 117). Gerechtigkeit lässt sich allgemein zunächst mithilfe des suum cuique-Prinzips erfassen: Gerecht ist eine Ordnung dann, wenn alle Akteure aus ihrer Perspektive das ihnen Zustehende erhalten haben. ${ }^{6}$ Allgemein lassen sich die Dimensionen globaler Gerechtigkeit mit Nancy Fraser (2008: 19; 2006) in den Kategorien Verteilung, Repräsentation und Anerkennung fassen.

Die Perspektive der internationalen Ethik bildet gleichsam den wissenschaftlichen Rahmen für die Untersuchung moralischer Kategorien und Gerechtigkeitsprinzipien auf globaler Ebene. Christoph Broszies und Henning Hahn (2010: 12) sprechen von einem Paradigmenwechsel, den die Debatte um globale Gerechtigkeit ausgelöst habe. So wurden die in der Gerechtigkeitstheorie zur Verfügung stehenden Begrifflichkeiten und Modelle mit dem Blick auf den Nationalstaat entwickelt und lassen sich von daher nicht ohne Weiteres auf die globale Ebene übertragen. John Rawls (2001; 1972), der »Vater « der modernen Gerechtigkeitsforschung, zeigte sich selbst zögerlich, seine auf der nationalstaatlichen Ebene entwickelten liberalen Gerechtigkeitsprinzipien auf die weltpolitischen Verhältnisse zu übertragen. In seinem »Recht der Völker« (1999) fragt Rawls nach den völkerrechtlichen Grundsätzen liberaler Außenpolitik. Hierfür entwickelt er ein zweistufiges Modell: Während das Wohlergehen der Bevölkerung und damit soziale Gerechtigkeit als partikulare Gerechtigkeitsprinzipien auf nationalstaatlicher Ebene zu realisieren sind, obliegt es vor allem den demokratischen Staaten, die institutionellen Voraussetzungen in globalem Maßstab zu schaffen. ${ }^{7}$ Für die internationale Ebene benennt Rawls (1999: 37) notwendige minimale und eher allgemeine Gerechtigkeitsprinzipien, wie etwa die wechselseitige Nichteinmischung (non-intervention), das Recht auf Selbstver-

6 Das suum cuique-Prinzip ist von Platon als Grundsatz der Verteilungsgerechtigkeit formuliert worden (Forst 2010: 31). Prinzipiell lässt es sich jedoch auch auf die anderen Dimensionen der Gerechtigkeit (Anerkennung, Verfahren) übertragen.

7 Da Rawls in seiner Konzeption von »Gerechtigkeit als Fairness« (2001) der politischen Ordnung der Demokratie Vorrang vor philosophischen Überlegungen der Gerechtigkeit einräumt, ist es für das »Recht der Völker« (1999) konsequent, ebenfalls Unterschiede in der politischen Ordnung von Gesellschaften zu berücksichtigen. Siehe dazu Goldstein (2012: 85). 
teidigung und die Achtung von Menschenrechten (Broszies/Hahn 2010: 43). ${ }^{8} \mathrm{Zu}-$ dem differenziert Rawls Staaten bzw. Völkergemeinschaften entlang ihrer politischen Verfasstheit. Liberale Demokratien können solche nicht-liberalen Staaten als völkerrechtlich gleichwertig anerkennen, die grundlegende Menschenrechte befolgen. ${ }^{9}$ Gegenüber den »burdened societies « (Rawls 1999: 63) besteht dann zumindest eine grundlegende Verpflichtung der Hilfeleistung.

Vertreter eines moralischen Kosmopolitismus argumentieren, dass eine solche "Arbeitsteilung «, wie Rawls sie vornimmt, in der heutigen interdependenten, globalisierten Weltpolitik nicht ausreicht und wir eine moralische Verpflichtung jenseits staatlicher Grenzen haben, jene Verteilungsungerechtigkeiten, die sich insbesondere in Gestalt von Armut und Unterernährung zeigen, zu beseitigen (Pogge 2007: 34; Beitz 1979: 128). Rawls wird neben seiner Akzeptanz illiberaler Regime als völkerrechtlich gleichwertige Subjekte von kosmopolitischer Seite auch vorgehalten, die eigenen, auf nationalstaatlicher Ebene in »Theory of Justice« (1972) gewonnenen, Gerechtigkeitsprinzipien, wie das elementarer Bürgerrechte der Freiheit und Chancengleichheit, in seiner Konzeption globaler Gerechtigkeit außer Acht gelassen zu haben (Moellendorf 2002: 15). Charles Beitz (1979: 144-153) überträgt Rawls' kontraktualistische Gerechtigkeitskonzeption auf die globalen Verhältnisse und argumentiert, dass die Realisierung eines globalen Freiheits- und Differenzprinzips auch im rationalen Interesse der Menschen weltweit sein müsste.

In den Konzeptionen einer internationalen Ethik und der globalen Gerechtigkeit finden sich verschiedene Varianten des Kosmopolitismus. Die Ursprünge der cosmopolis gehen zurück bis in die griechische Antike: Die Stoiker entwickelten mit der Idee des Weltbürgertums Vorstellungen der historischen Kontingenz staatlicher Grenzen (Beardsworth 2011: 17). ${ }^{10}$ Der moralische Kosmopolitismus ${ }^{11}$

8 Rawls (1999: 29) selbst spricht in seiner Abhandlung bewusst vom Recht der Völker und versteht darunter konstitutionelle Demokratien, die ihre Beziehungen mittels Gerechtigkeitsprinzipien regulieren. Während BürgerInnen aus seiner Sicht rational handeln, macht er in souveränem staatlichen Handeln expansionistische Bestrebungen aus - dieses vermag unter Umständen zu hegemonialen Kriegen führen.

9 Rawls (1999: 63) bezeichnet diese Gesellschaften als »decent hierarchical societies« und differenziert sie von »outlaw states « und »burdened societies«.

10 Allerdings entsprang aus dieser Form des ethischen Weltbürgertums noch keine staatszentrierte Gerechtigkeitskonzeption, die sich erst mit dem Aufkommen eines staatszentrierten Rechtsdenkens entwickeln konnte (Broszies/Hahn 2010: 13).

11 Gillian Brock (2009: 11-13) verweist auf die Bandbreite kosmopolitischer Gerechtigkeitsansätze, etwa auf utilitaristische oder auch Kantianische Ansätze. Beardsworth (2011:21-47) differenziert dagegen zwischen dem kulturellen, moralischen, normativen, institutionellen, politischen und rechtlichen Kosmopolitismus. Siehe dazu auch G. Brock/Brighouse (2005) sowie Tan (2004: 10-12). 
knüpft daran an und nimmt eine »common human community« (Shapcott 2010: 15) auch jenseits nationalstaatlicher Grenzen an. Diesem Ansatz zufolge gelangen Menschen auch auf globaler Ebene zu gemeinsamen normativen Maßstäben, wie Moralund Gerechtigkeitsüberzeugungen, weil sie im Sinne Kants alle gleichermaßen mit Vernunft und Rationalität ausgestattet sind. Dagegen vertreten institutionelle Kosmopolitisten die Auffassung, dass globale Gerechtigkeitsprinzipien nicht individuell, sondern vor dem Hintergrund internationaler Missstände von globalen Ausgrenzungs-, Ausbeutungs- und Herrschaftsstrukturen zu begründen seien, um den Effekten solcher strukturellen Ungerechtigkeiten zu begegnen (Broszies/Hahn 2010: 12). Thomas Pogge (2002 a: 14) überträgt deshalb Rawls' Differenzprinzip auch auf die internationale Ebene: Ökonomische Vorteile sind nur solange gerechtfertigt, solange auch die Benachteiligten davon profitieren. Er argumentiert für Verteilungsgerechtigkeitsprinzipien auf der Grundlage minimalmoralischer Schadensvermeidung: Gerade weil die bestehenden weltwirtschaftlichen Institutionen zur Armut beitrügen, leite sich daraus eine Verantwortung der »global rich « für die Armen ab, die verantwortlichen Institutionen so zu verändern, dass solche Ausbeutungsstrukturen beseitigt würden.

Pogge (2002 a: 125) zufolge lässt sich die kosmopolitische Moral allgemein anhand von drei Kriterien zusammenfassen: Individualismus, Universalismus und Allgemeinheit der Verpflichtungen. ${ }^{12}$ Wenn Individuen aufgrund ihrer Staatsangehörigkeit schlechtere Lebenschancen haben, folgt im Kosmopolitismus eine moralische Verpflichtung, die sich Menschen als Menschen mit dem Ziel schulden, Leid $\mathrm{zu}$ vermeiden und aktiv gegen Unrecht und Ungleichheit vorzugehen ( $\mathrm{Lu} 2000$ : 263). Individuelle Menschenrechte erhalten Priorität gegenüber den Prinzipien staatlicher Souveränität (Pogge 1992: 58).

Kosmopolitisten sind überzeugt, dass sich universelle Gerechtigkeitsprinzipien ermitteln lassen, die jedem Menschen gleichermaßen zustehen. Uneinigkeit besteht jedoch über die Reichweite und den Umfang solcher Menschenrechte (Pogge 2002 b: 158) - vor allem inwieweit und in welchem Umfang sie ökonomische undoder soziale Gerechtigkeit umfassen (Caney 2005: 102-147; Moellendorf 2002: 36). Martha Nussbaum (2006) entwirft den sogenannten »Fähigkeitenansatz« ( $c a-$ pabilities), der umfassende Forderungen an das Prinzip der Verteilungsgerechtigkeit stellt: Je gravierender Ungleichheiten zwischen den Menschen ausfallen, desto

12 Caney (2010: 148) nennt diese Form des Kosmopolitismus auch »justice-based«, weil es ihm um die Feststellung globaler Gerechtigkeitsprinzipien gehe. 
komplexer müssten die Forderungen nach Gleichheit ausfallen. ${ }^{13}$ Nussbaums Liste der grundlegenden menschlichen Fähigkeiten ist nahezu deckungsgleich mit der Allgemeinen Erklärung der Menschenrechte, weshalb sie ihre Gerechtigkeitstheorie auch als »Menschenrechtsansatz« bezeichnet (Nussbaum 2006: 392). Sie hat innerhalb des Kosmopolitismus eine der gehaltvollsten Gerechtigkeitskonzeptionen entworfen, die auch Aspekte sozialer Gerechtigkeit umfasst - allerdings ist dieser Fähigkeitenansatz selbst unter Kosmopolitisten umstritten.

Die kommunitaristische Kritik am Kosmopolitismus entzündet sich unter anderem auch daran, dass dieser den profunden kulturellen und normativen Pluralismus in der Welt übersehe. Gerade deshalb sei die kosmopolitische Idee der menschlichen Gleichheit eine elementare Ungerechtigkeit und als Grundlage für die Annahme globaler Gerechtigkeitspflichten zu schwach. Gerechtigkeit entstehe im Kontext der jeweiligen Gemeinschaft und falle unterschiedlich aus, wenn man etwa nach China oder in die Vereinigten Staaten blicke (Walzer 2006; Brown 2002: 92-93; 1992). Kommunitaristen vertreten die Auffassung, dass nur Menschen einer abgrenzbaren Gemeinschaft Subjekte von Gerechtigkeit sein können, denn nur dieser Personenkreis lebe in einer für Gerechtigkeit konstitutiven Beziehungsform zusammen. Gerechtigkeit ist diesem Ansatz zufolge also von vornherein kontextual und partikular angelegt und somit nicht von globaler Gültigkeit (Miller 2007: 263; 2005; Walzer 1994: 83). Kommunitaristen verweisen auf die Relevanz von Grenzen, an denen der Anspruch von (sozialer) Gerechtigkeit ende (Nagel 2005).

Bei aller Betonung der Differenzen identifizieren auch Kommunitaristen ein wenn auch schwaches - universalistisches Element. Menschen aus verschiedenen Kulturen vollziehen im globalen Raum eine wechselseitige Annäherung - daraus entstehe eine neue, transnationale Gemeinschaft, in der sich eine gemeinsame, wenngleich auch nur dünne Schicht an Handlungsnormen herausbilden könne. So lassen sich auch moralische und noch konkreter humanitäre menschenrechtliche Pflichten jenseits gemeinschaftlicher Grenzen feststellen. In diesem Zusammenhang wird auch auf globaler Ebene ein Minimalmoralismus, unter anderem in Form der Schadensvermeidung, anerkannt (Shapcott 2010: 59; Miller 1999: 197; Walzer 1994: 103; Hutchings 1999). Kommunitaristen würden aber die Frage der Verantwortung jenseits staatlicher Grenzen weitaus restriktiver beantworten als Vertreter des Kosmopolitismus. Im folgenden Kapitel soll nun der Frage nachgegangen wer-

13 Nussbaum (2006: 2) kritisiert andere Theorien sozialer Gerechtigkeit dafür, dass sie stets den Nationalstaat zum Ausgangspunkt ihrer Überlegungen machten. Eine Theorie globaler Gerechtigkeit müsse neue theoretische Strukturen entwickeln, um der globalen Ungleichheit zu begegnen. 
den, inwieweit sich daraus auch moralische Verpflichtungen ableiten lassen, die den Einsatz von militärischer Gewalt rechtfertigen.

\section{Solidarität jenseits staatlicher Grenzen? Kosmopolitische Rechtfertigungsgründe für die militärische Intervention}

In der Debatte um eine internationale Ethik des Einsatzes von Gewalt (use of force) geht es um die Frage der Reichweite und Handlungsimplikationen der Verantwortung von Staaten jenseits der eigenen staatlichen Grenzen. Haben andere Staaten im Fall von Menschenrechtsverletzungen die moralische Verpflichtung, notfalls auch militärisch zu intervenieren? So wie Rawls als »Vater« der Gerechtigkeitsdebatte begriffen wird, kommt Michael Walzer dieser Status in Fragen des »Gerechten Krieges« zu. Walzer (1977: 54) argumentiert prinzipiell kommunitaristisch und verteidigt die Nichteinmischung in die inneren Angelegenheiten der Staaten sowie das Prinzip der Nichtintervention. ${ }^{14}$ In seiner Argumentation folgt er der liberalen Überzeugung John Stuart Mills und sieht einen starken Zusammenhang zwischen der äußeren Souveränität von Staaten und der staatlichen Verpflichtung, im Inneren für das individuelle Wohlergehen der eigenen Bürger zu sorgen. Der gerechte Grund für den Gewalteinsatz ist die Selbstverteidigung im Fall einer äußeren Aggression, weil damit nicht nur das staatliche Selbstbestimmungsrecht, sondern auch die individuellen Rechte der Bürger bedroht würden. Konsequenterweise impliziere staatliche Selbstbestimmung auch, dass im Fall von Bürgerkriegen eine Einmischung von außen unterbleiben sollte. ${ }^{15}$ Walzer (1977: 90) nennt drei mögliche Einschränkungen des Souveränitätsprinzips und Rechtfertigungsgründe für Interventionen: erstens Sezessionen oder nationale (postkoloniale) Befreiungsbewegungen; zweitens Konter-Interventionen und drittens humanitäre Interventionen. Bei schweren Menschenrechtsverletzungen hält er eine Revision des »legalist paradigma « und der Prinzipien der Nichteinmischung und Nichtintervention für gerechtfertigt, wenn eine angemessene Aussicht auf einen Erfolg der Intervention be-

14 Erskine (2000: 583-588) weist Walzer nach, dass er in seiner Argumentation durchaus kosmopolitische Züge aufweist. Sein Beispiel vom »nackten Soldaten«, der im Feind den Menschen erkennt und sich dem Schießbefehl verweigert, ist für sie Beweis, dass auch Walzer der Idee einer »common humanity « jenseits staatlicher Grenzen nachspürt.

15 Hierfür verweist er erneut auf die millsche Analogie zwischen der Kultivierung individueller Freiheit und nationaler Selbstbestimmung: »Members of a political community must seek their own freedom, just as the individual must cultivate his own virtue. They cannot be set free, as he cannot be made virtuous, by any external force (Walzer 1977: 87). 
steht (Walzer 1977: 106-107). ${ }^{16}$ In späteren Werken sieht Walzer (2003: 40; 2002 : 938) die Lehre vom »Gerechten Krieg « in ernster Gefahr, da sie sowohl von Seiten der Politik wie auch des Militärs verstärkt missbräuchlich verwendet würde. ${ }^{17}$

Neuere Konzeptionen zur Lehre des »Gerechten Krieges« argumentieren dagegen primär kosmopolitisch. ${ }^{18}$ Sie verweisen auf die veränderte weltpolitische Ausgangslage und neue Kriegsformen, die eine zeitgemäßere Interpretation der Lehre vom »Gerechten Krieg « notwendig machten. Walzers Kriegstypologie sei überholt; statt staatlicher Sicherheit müsse menschliche Sicherheit zum Fokus einer zeitgemäßeren Interpretation des »Gerechten Krieges« gemacht werden (Hudson 2009: 44). So argumentiert beispielsweise Patrick Hayden (2005: 158-160) auf der Grundlage des moralischen und normativen Kosmopolitismus, individuelle moralische Rechte seien bereits in den Normen und Regeln des bestehenden Menschenrechtsregimes verankert und diese Institutionalisierung würde sich auch in anderen Politikfeldern fortsetzen. ${ }^{19}$ Auf eine ähnliche Begründung greift Habermas in seinen Überlegungen zum Kosovo-Konflikt zurück, wenn er davon spricht, dass die Intervention »einen Sprung auf dem Wege des klassischen Völkerrechts der Staaten zum kosmopolitischen Recht einer Weltbürgergesellschaft bedeuten « könnte (Habermas 1999 b). ${ }^{20}$

Simon Caney (2005: 194) kritisiert Walzer dafür, dass dieser Souveränität und Staatenrechte über den Schutz individueller Menschenrechte stelle. Walzers staatszentrierte Ordnung lasse die Möglichkeit weitgehender innerer Repressionen gegen die eigene Bevölkerung zu und versäume es, Individuen in angemessener Weise vor

16 In diesem Fall verletzt der Staat, der Gewalt gegen seine Bevölkerung ausübt, die individuellen Rechte seiner BürgerInnen und verwirkt damit seine souveränen Grundrechte. Diese Idee, dass staatliche Souveränität kontraktualistisch eingeschränkt ist und staatliche Autorität die Sicherheit der BürgerInnen beinhaltet, lässt sich ideengeschichtlich schon bei Jean Bodin und Thomas Hobbes finden. Die Idee der »popular sovereignty« ist beispielsweise auch in der amerikanischen Unabhängigkeitserklärung von 1776 verankert worden. Siehe dazu Glanville (2010: 240), Jahn (2012: 43-53) sowie Nardin (2002).

17 Walzer bemängelt hierbei vor allem die Entgrenzung der gerechten Gründe bis hin zum »regime change « und die Vernachlässigung des ius in bello gerade auch im Hinblick auf den Schutz von Nicht-Kombattanten.

18 Beardsworth (2011:29-40) benennt neben dem moralischen auch den normativen sowie den rechtlichen (legal) Kosmopolitismus.

19 Habermas (2004: 160-165) verweist auf die Vielzahl neuer Institutionen, die für ihn einen wachsenden Korpus des Weltbürgerrechts ausmachen. Als Beispiel nennt er den Internationalen Strafgerichtshof, der individuelle Rechte jenseits staatlicher Grenzen stärke.

20 Beardsworth (2011:37) sieht Habermas als den Prototypen des rechtlichen Kosmopoliten. Zu Habermas' moralischem Dilemma siehe Fn. 33. 
Übergriffen durch Unrechtsregime zu schützen. ${ }^{21}$ Der kosmopolitische Standpunkt würde vielmehr nach einer Revision des ius ad bellum-Prinzips des »gerechten Grundes « (just cause) verlangen (Fabre 2008: 965). Kosmopolitisten verweisen auf die Bedeutsamkeit der moralischen Legitimität von Staaten: Neben der Wahrung von Menschenrechten muss es Staaten auch darum gehen, ihre Herrschaft über die Zustimmung der eigenen Bevölkerung zu sichern. Für Darrel Moellendorf (2002: 161) ist es nicht nur eine Frage der Zustimmungsfähigkeit staatlichen Handelns, sondern auch der inneren Verfasstheit von Staaten. Deshalb zweifelt er am gerechten Grund der Intervention gegen den Irak im Jahre 1991: Die Intervention brachte seiner Meinung nach keine nachhaltigen Verbesserungen für die Kuwaiter, da das Unrechtsregime auch danach noch weiterexistiert habe. Caney (2005: 204) erweitert die kosmopolitische Auslegung des gerechten Grundes: Die Intervention ist dann gerechtfertigt, wenn ein Krieg einen gerechteren Staat im Sinne der kosmopolitischen Prinzipien der Gewährleistung bürgerlicher Grundfreiheiten hervorbringen würde. ${ }^{22}$ Fernando R. Tesón (2005: 2; 2003) hält humanitäre Interventionen für angemessen, wenn sich dadurch Unrechtsregime oder innerstaatliche Anarchie beseitigen lassen (Bellamy 2006: 202). In einer solchen selektiven kosmopolitischen Lesart des »Gerechten Krieges« würde sich der externe, militärisch erzwungene demokratische Regimewechsel moralisch rechtfertigen lassen, auch wenn er gegen die Normen der Nichtintervention und Nichteinmischung der UN-Charta und somit gegen bestehendes Völkerrecht verstoßen würde.

Kimberly A. Hudson (2009: 55) verweist auf die im Rom-Statut identifizierten Kriegsverbrechen, die einer Neuinterpretation der gerechten Gründe für eine bewaffnete Intervention zugrunde gelegt werden könnten. Sie betont allerdings auch, dass mit dem gerechten Grund nur der Typ des Verbrechens benannt sei und die übrigen Kriterien der Lehre vom »Gerechten Krieg « ${ }^{23}$ ebenfalls in Gänze in Anschlag gebracht werden müssten, um eine militärische Intervention zu rechtfertigen

21 Caneys Kritik richtet sich diesbezüglich nicht nur gegen Walzer, sondern auch gegen Nardin (1983).

22 Caney (2005: 240-244) selbst benennt eine ganze Reihe von Gründen, weshalb Interventionen und im besonderen humanitäre Interventionen - oftmals wenig Aussicht auf (nachhaltigen) Erfolg haben (mangelnde Kenntnis der lokalen Gegebenheiten; Eigeninteresse statt altruistischer Motive der intervenierenden Staaten; Widerstand gegen die Intervention durch die Bevölkerung; millsche Überlegungen: politische Systeme nur dann nachhaltig stabil, wenn die Bevölkerung dahinter steht).

23 Die Kriterien des Gerechten Krieges umfassen im Hinblick auf das ius ad bellum (Recht zum Krieg) neben dem gerechten Grund auch den Aspekt des Erfolgs, der legitimen Autorität, der richtigen Intention und des letzten Mittels (last resort). Mit Blick auf das ius in bello (Recht im Krieg) werden die Kriterien der Angemessenheit der Mittel und des Schutzes von Nicht-Kombattanten (discrimination) angeführt. Siehe dazu Bellamy (2006: 127). 
(Hudson 2009: 59). Legt man die Kriterien der Lehre vom »Gerechten Krieg« in Gänze als Maßstab zugrunde, dann kommen Autoren selbst im Fall des NATOEinsatzes im Kosovo zu dem Schluss, dass die Intervention auch moralisch nicht legitim war (Mayer 1999: 317; Merkel 2004: 130; Haspel 2002: 215). ${ }^{24}$

Zusammengefasst zeigt sich, dass die Lehre des »Gerechten Krieges« im Kosmopolitismus verstärkt zur Anwendung kommt. Diese neuere kosmopolitische Interpretation vom »Just War« lassen sich mittels vier zentraler Prinzipien zusammenfassen: 1.) Aus dem Individualismus, woraus folgt, dass die Rechte der Menschen zu schützen sind (Elshtain 2003: 66); 2.) Daraus, dass Staaten und ihre politischen Institutionen die Menschenrechte ihrer Bürger wahren müssen, zumindest aber nicht verletzen dürfen; 3.) Daraus ergibt sich eine moralische Verpflichtung anderer Staaten, Menschenrechte auch jenseits der eigenen Staatsgrenzen zu schützen; 4.) Die militärische Intervention muss erfolgversprechend im Sinne des Menschenrechtsschutzes sein (Caney 2005: 232-233; Moellendorf 2002: 142) ${ }^{25}$ Innerhalb des Kosmopolitismus lassen sich jedoch durchaus unterschiedliche Positionen der moralischen Rechtfertigung des Gewalteinsatzes ermitteln. Insgesamt lässt sich feststellen, dass dort, wo die Kriterien des »Gerechten Krieges « selektiv verwendet werden, eine Tendenz zur Entgrenzung eines gerechtfertigten Gewalthandelns zu beobachten ist.

\section{Solidarität jenseits staatlicher Grenzen? Rechtfertigungen aus der Perspektive der Theorien Internationaler Beziehungen}

Ähnlich wie in der IPT lassen sich auch in den Theorien der IB eine ganze Bandbreite von unterschiedlichen Ansätzen identifizieren, die Aussagen zur Verantwortlichkeit von Staaten jenseits der eigenen Grenzen im Hinblick auf die Rechtfertigung militärischer Interventionen machen. ${ }^{26}$ In der IB-Debatte bildet das Spannungsfeld zwischen individuellen Rechten bzw. Menschenrechten und staatlicher Souveränität

$24 \mathrm{Zu}$ diesem Schluss gelangt auch Hudson (2009: 157). Zwar sei ein gerechter Grund auf der Basis der im Rom-Status ausgewiesenen Kriegsverbrechen gegeben, doch seien die Kriterien »last resort, proportionality and likelihood of success « nicht gewährleistet gewesen. Die oben zitierten Autoren begründen ihre Ablehnung primär mit der Missachtung des ius in bello. Aber auch Kriterien des ius ad bellum seien nicht erfüllt gewesen, beispielsweise das Kriterium des letzten Mittels oder auch der legitimen Autorität.

25 Manche Kosmopoliten, wie Beitz (1979: 390), sehen gerade im vierten Punkt ein Problem und stehen humanitären Interventionen von daher eher skeptisch gegenüber.

26 Der Fokus der Auswahl aus den verschiedenen Theorien der IB liegt auf den normativen Ansätzen. Häufig wird darunter auch der Sozialkonstruktivismus subsumiert - da er allerdings keine substanziellen ethischen Aussagen macht, wird er hier im Sinne Hoffmanns (2009) nur als möglicher Ansatz der Diskursethik reflektiert. Siehe dazu Kapitel 5. 
den Rahmen für die Debatte zwischen den und innerhalb der verschiedenen Denkschulen. Am deutlichsten artikuliert findet sich diese Differenz in den zwei Ansätzen der Englischen Schule. In deren pluralistischer Lesart bilden Staaten Institutionen mit dem Ziel, Krieg und Gewalt zwischen ihnen zu minimieren, nicht aber zu eliminieren. Staatliche Souveränität und das Prinzip der Nichteinmischung bleiben ordnungsstiftende Prinzipien - Gerechtigkeit ist im pluralistischen Ansatz Aufgabe der jeweiligen Nationalstaaten (Bull 1977; Dunne 1998; Jackson 2000; Wheeler 1992: 477). Dennoch kann über moralische und rechtliche Normen eine Begrenzung von menschlichem Leid auch auf internationaler Ebene erreicht werden. Solche Normen sind in grundlegenden harm conventions, etwa im internationalen Völkerrecht, realisiert. Neuere Ansätze der Englischen Schule wollen über die universale Definition solcher harm-Prinzipien eine konsensuale, minimale Gerechtigkeitsethik für die internationalen Beziehungen entwickeln. ${ }^{27}$ Die Grenze der Solidarität ist für Andrew Linklater jedoch im Fall von Gewaltanwendung zum Schutz von Menschenrechten oder bei humanitären Interventionen erreicht - beides dürfe keine »basic norm of world politics « werden (Linklater 2002: 147; 2006: 334).

Vertreter des solidarischen Ansatzes der Englischen Schule befürworten weitergehende Grundsätze. ${ }^{28}$ Die Konzeption von Ordnung und Gerechtigkeit in der pluralistischen Variante der Englischen Schule ist für Solidaristen Ausdruck eines überkommenen weltpolitischen Verständnisses aus Zeiten des Ost-West-Konflikts (Hurrell 2003: 26). Ihnen zufolge weisen die normativen Strukturen der internationalen Gemeinschaft inzwischen deutliche Zeichen größerer Solidarität und geteilter Gerechtigkeitsüberzeugungen auf (Buzan 2004: 141; Wheeler 2000: 12). ${ }^{29}$ Das Ordnungsprinzip der staatlichen Souveränität beinhalte die globale Verantwortung des Schutzes aller Menschen. Einer solchen kosmopolitischen Moral entspräche als Institution die humanitäre Intervention, die notfalls auch gegen den Willen der herrschenden Regierung zum Schutz der Menschen(rechte) veranlasst werden müsse (Roennfeldt 1999: 141-168; L. Brock 2002: 214). Die Staatenräson der Nichteinmischung und Kriegsverhütung wird somit kosmopolitischen Gerechtigkeitsüberzeugungen untergeordnet (Dunne/Wheeler 2004: 18). Gewaltanwendung wird le-

27 Auf diese Weise lässt sich für Linklater $(2002 ; 2006)$ auch die Differenz zwischen Pluralismus und Solidarismus sowie Kommunitarismus und Kosmopolitismus überwinden. Die Vermeidung menschlichen Leids dient für ihn als Prinzip, über das sich eine Verantwortung jenseits staatlicher Grenzen ableiten und gleichsam in eine minimale Gerechtigkeitsethik überführen lässt.

28 Buzan (2004: 149) weist zu Recht darauf hin, dass das Argument des Menschenrechtsschutzes schon in den Anfängen der Englischen Schule eine wichtige Rolle gespielt hat, beispielsweise bei Vincent (1986: 114).

29 In dieser Argumentation finden sich auffällige Übereinstimmungen mit dem von Beardsworth (2011: 29-34) entwickelten normativen Kosmopolitismus. 
gitim, wenn sie dem Schutz des Individuums dient. Neben Genozid und Völkermord rechtfertigen auch Massenvertreibung und Staatszerfall die Solidarität der internationalen Staatengemeinschaft (Wheeler 2000: 34). Allerdings präferieren die Solidaristen der Englischen Schule andere Formen der politischen Steuerung jenseits der militärischen Gewaltanwendung: Präventiver Diplomatie kommt im Hinblick auf Staatszerfall und Menschenrechtsverletzungen eine besondere Rolle zu (Dunne/ Wheeler 2004: 20).

Liberale Internationalisten argumentieren zunächst ähnlich wie die Solidaristen der Englischen Schule. ${ }^{30}$ Basierend auf der liberalen politischen Philosophie haben Staaten diesem Ansatz zufolge die Verpflichtung, das Wohlergehen, die Rechte und die Gleichheit ihrer Bürger zu sichern (Bishai 2004: 52). Zugleich hat sich das Prinzip staatlicher Souveränität nach dem Verständnis dieser Denkschule verändert: Statt staatlicher Autorität steht jetzt die staatliche Verantwortlichkeit für den Schutz der Menschen auch außerhalb der eigenen Grenzen an erster Stelle (Evans/Sahnoun 2002: 101). Dieser »neue Humanitarismus « versteht sich als Teil einer liberalen Weltordnung, in dem der Schutz der Menschenrechte - die Wahrung von Freiheit und Rechten der Bürger - zum starken Argument für verschiedene Formen humanitärer Hilfe von Entwicklungszusammenarbeit bis hin zur militärischen Intervention wird (Barnett 2005: 733; Mills 2005). Zum Wesen dieses »neuen Humanitarismus « gehört auch, dass handelnde Akteure (Nichtregierungsorganisationen, Staaten) bewusst Partei für eine Seite ergreifen und die klassischen Prinzipien der humanitären Hilfe, wie Neutralität und Gewaltlosigkeit, dabei außer Acht lassen (Shapcott 2010: 133).

In der Debatte um die Frage des präventiven Gewalteinsatzes vertreten Allen Buchanan und Robert O. Keohane (2005: 4-5) als liberale Internationalisten die Auffassung, dass schon die Gefahr der Verletzung elementarer Menschenrechte präventives militärisches Handeln der Staaten rechtfertigt. Obwohl diese Form der Gewaltanwendung ihre Legitimität eigentlich nur im Rahmen vorhandener Institutionen gewinnt, kann »illegale Gewalt«, also der bewusste Verstoß von Staaten gegen bestehende völkerrechtliche Konventionen, unter Umständen den entscheidenden Anstoß für notwendige normative Veränderungen solch überkommener Institutionen bedeuten und erscheint in diesem Denken deshalb als legitim (Buchanan 2003: 134). In zentraler Verantwortung steht dabei eine Koalition demokratischer Staaten, denen aufgrund ihrer inneren Verfasstheit eine besondere »moral reliability« im Hinblick auf die Durchsetzung kosmopolitischer Prinzipien zugestanden

30 Ein guter Überblick über die Argumentationen liberaler Internationalisten findet sich auch bei Lang (2010: 329-331). 
wird (Buchanan/Keohane 2004: 19; Tesón 2003). ${ }^{31}$ Andere Vertreter des liberalen Internationalismus betrachten nicht nur die Demokratie, sondern auch den Großmachtstatus (beispielsweise der Vereinigten Staaten) als entscheidende Legitimation, den Schutz von Menschenrechten global durchzusetzen, da die globalen Institutionen der kollektiven Entscheidungsfindung ihnen zufolge noch nicht hinreichend ausgestaltet sind (Elshtain 2003: 74).

Der liberale Internationalismus bekennt sich nicht nur zu den Prinzipien des Humanitarismus, des Schutzes individueller Menschenrechte bei gleichzeitiger Inkaufnahme der Verletzung der Prinzipien staatlicher Souveränität und Nichteinmischung, sondern auch zur Gewaltanwendung gegenüber dem »ungerechten Feind «. Nach 9/11 sind die nationalen Interessen der Demokratie und die Ziele des Humanitarismus nach Überzeugung liberaler Internationalisten weiter zusammengewachsen (Feinstein/Slaughter 2004: 149). Kosmopolitische Moral- und Gerechtigkeitsüberzeugungen dienen dabei als starke argumentative Rechtfertigung von Gewalt: Der »Krieg gegen den Terror « wird zur moralischen Mission der »zivilisierten Welt« gegen das »unzivilisierte Böse« (Burke 2004: 334). Das Ziel der Demokratisierung und Zivilisierung, das notfalls auch mithilfe militärischer Gewalt durchgesetzt werden soll, wird dabei zum zentralen handlungsanleitenden Prinzip (Hobson 2008). So argumentieren Lee Feinstein und Anne-Marie Slaughter (2004: 137) selbst dann für präventive Gewalt im Umgang mit nicht-demokratischen Staaten, die nach Kernwaffen streben, wenn diese Mitglieder des nuklearen Nichtverbreitungsvertrages sind. Im Mittelpunkt dieses liberalen Internationalismus steht immer wieder die Konstruktion des »Anderen«, des außerhalb der »menschlichen Gemeinschaft« stehenden »Bösen«, das dem Aufbau einer gerechten internationalen Gesellschaft und Weltordnung zuwiderhandelt (Moses 2010: 31; Desch 2007).

\section{Kritische Ansätze und der Umgang mit differenten Gerechtigkeitsvorstellungen}

Aus Sicht ihrer Kritiker übersehen die »Advokaten liberaler Hierarchie« die Diskrepanzen zwischen moralischem Ideal und politischer Realität (Reus-Smit 2005: 83). So strebten Demokratien einerseits danach, universell erachtete Standards (Demokratie, Menschenrechte) durchzusetzen, verweigerten sich jedoch andererseits der konsequenten Einschränkung ihrer Souveränität und Autonomie im Rahmen des

31 Smith (1992: 212) und andere Vertreter der politischen Theorie verweisen darauf, dass es sich dabei um eine falsch verstandene Interpretation des Liberalismus handele. Dieser sei gerade im Hinblick auf die Frage des Interventionismus und Prinzipien internationaler Gewaltanwendung bestenfalls ambivalent. 
Völkerrechts (Geis et al. 2007 a: 29; L. Brock 2007; Deitelhoff 2007). Die eigenen liberalen Normen und Werte würden zum zivilisatorisch-missionarischen Friedensprogramm gegenüber dem »ungerechten Feind « (Geis et al. 2007 b: 83). In der Begründungsrhetorik des »Krieges gegen den Terror« und der Bekämpfung des »ungerechten Feindes« habe sich auch die humanitäre Überzeugung gefunden, zum Wohle der unterdrückten Bevölkerung zu handeln. Ein solcher moralisch begründeter Humanitarismus führe jedoch zur Entgrenzung des Gewalthandelns (Farer 2003: 55; Burke 2004: 331; 2005). ${ }^{32}$ Der aus humanitären Gründen geführte $» k o s-$ mopolitische Krieg « werde gleichsam zur konstitutiven Bedingung, um Frieden zu ermöglichen und die internationale Rechtsordnung im Sinne des Menschenrechtsschutzes zu stärken (Jabri 2007 a: 64). ${ }^{33}$ Patricia Owens (2007: 131) kritisiert Habermas dafür, den Krieg als politisches Mittel dem moralischen Ziel - der Institutionalisierung von Weltbürgerrechten - unterzuordnen und somit zu legitimieren. ${ }^{34}$ Kritik richtet sich auch gegen die Versuche, mithilfe der »Just War Theory « Krieg zu rechtfertigen und gleichzeitig in seinem Ausmaß zu begrenzen. Vielmehr scheinen die beständigen Versuche der Fixierung universaler Gerechtigkeitsprinzipien im Umgang mit Gewalt zu größerer globaler Ungerechtigkeit beizutragen (Campbell/Shapiro 1999: x-xi).

Die Bedenken gegenüber solchen Formen des »liberalen Imperialismus« sind gerade im globalen Süden groß (Purdy 2005: 323; Barnett 2005: 734; Duffield 2001). Im indischen Verständnis sind dem Staat im Zuge der Dekolonialisierung emanzipatorische Aufgaben zugekommen - staatliche Souveränität und Nichteinmischung werden deshalb als Institutionen freier Menschen und als Gerechtigkeitsprinzipien begriffen (Bajpai 2003: 260). Aus dieser Perspektive dienen Menschenrechte und deren proaktive Durchsetzung dem Westen als Legitimation von Gewalt und Verbrämung eigener strategischer Interessen und helfen dabei, sich gegen alle

32 Owens (2007) rekurriert dabei auf die Philosophie Hannah Arendts und äußert sich kritisch zur Frage von Gewaltlegitimation und Intervention. Werde Gerechtigkeit zum Endziel, gerieten die »Mittel« häufig außer Acht. Owens zitiert Arendt: »By applying the absolute - justice, for example, or the ideal in general [...] to an end, one first makes unjust, bestial actions possible, because the rideal<, justice itself, no longer exists as a yardstick, but has become an achievable, producible end within the world « (Owens 2007: 145-146).

33 Sowohl Owens als auch Jabri verweisen dabei auf Habermas' Dilemma der Menschenrechtspolitik. In seinem Zeit-Artikel zum Kosovo-Konflikt schreibt er hierzu: »Vielfach ist sie [die Menschenrechtspolitik; Anm. SW] aber angesichts des unterinstitutionalisierten Weltbürgerrechts zum bloBen Vorgriff auf einen künftigen kosmopolitischen Zustand, den sie zugleich befördern will, genötigt« (Habermas 1999b). Gleichzeitig artikuliert er seine Zweifel an der Kriegsführung der NATO und kritisiert die Folgen für die Zivilbevölkerung.

34 Schmidt (2004: 253) moniert an Habermas' Begründung der Kosovo-Intervention, dass dieser auf die diskursethische Anlage einer Entwicklung solcher weltbürgerlichen Rechte verzichtet habe. 
Forderungen nach globaler Verteilungsgerechtigkeit und sozialer Gerechtigkeit zu immunisieren (Hurrell 2003: 41; Mutua 2001). Auch innerhalb Europas sind Menschenrechte nicht Teil der angestammten Kultur und Identität, sondern gegen eigene Traditionen und kulturellen Widerstand als Teil des Modernisierungsprozesses hart erkämpft worden (Senghaas 1998: 36). Aus der postkolonialen Perspektive wird jedoch genau dieser Modernisierungsprozess zum Problem für eine Konzeption universaler Gerechtigkeit, weil im Zuge des Kolonialismus und der Dekolonialisierung Exklusionsmechanismen und strukturelle Gewaltverhältnisse entstanden sind, die bis heute nachwirken und vom Westen nur unzureichend anerkannt werden (Jabri 2007 b: 721; 2007 a). Der historisch-genealogische Blick auf die Fundamentalnorm der Souveränität hilft zu dechiffrieren, warum zahlreiche Staaten des globalen Südens die Prinzipien der Nichteinmischung, der territorialen Selbstbestimmung und der Nichtintervention als unhinterfragbare Gerechtigkeitsauffassungen begreifen (Jackson 1990: 32-49). ${ }^{35}$

Ein möglicher Ausweg aus diesem Dilemma unterschiedlicher Gerechtigkeitsauffassungen bietet die Diskursethik. Aus der Perspektive der IPT lassen sich Unterschiede in Gerechtigkeitsauffassungen über angemessene Verfahren und faire Verständigung auflösen (Hutchings 2010: 43). Zu ähnlichen Schlüssen gelangen auch sozialkonstruktivistische Ansätze in den IB. Zwar lassen sich aus den Prämissen des Sozialkonstruktivismus selbst keine substanziellen ethischen Grundsätze ableiten, doch eignet er sich insbesondere dafür, eine Diskursethik zu entwickeln (Hoffmann 2009: 243). ${ }^{36}$ Zahlreiche Studien knüpfen deshalb an Habermas' Theorie des kommunikativen Handelns an und verbinden die Frage der Legitimität mit der Funktionalität deliberativer Verfahren (Müller 2008; Risse 2007; Deitelhoff 2006; Lynch 2000). Ziel im Umgang mit Verschiedenheit müsse es dementspre-

35 Jackson (1990: 32-49) beschreibt in einer teils ideengeschichtlichen, teils historisch-soziologischen Genealogie die unterschiedlichen Dimensionen von Souveränität, die eng verbunden waren mit der Entstehung der internationalen Beziehungen zwischen den Staaten. So war Souveränität bei Mill eng mit dem europäischen Staatsverständnis und Zivilisationsstandard verknüpft. Außereuropäische Staaten entsprachen diesen Kriterien nicht, somit musste ihnen das Recht auf Selbstbestimmung und souveräne Gleichheit vorenthalten bleiben. Ein solcher »standard of civilization «, wie Gong (1984) ihn bezeichnet, stand auch Pate für die Rechtfertigungsrhetorik europäischer Kolonisierungspolitik. Diese Konzeption der »positive sovereignty « wurde erst im Zuge der Dekolonialisierung durch das Konzept der »negative sovereignty « und den in der UN-Charta kodifizierten Prinzipien der Nichteinmischung und territorialen Integrität ersetzt. Siehe dazu auch Gong (1984).

36 Zwischen Sozialkonstruktivisten und Vertretern normativer Ansätze ist eine Debatte über Gemeinsamkeiten und Unterschiede entstanden. Auslöser war der Sammelband von Price (2008), der die Spannbreite sozialkonstruktivistischer Ansätze im Hinblick auf die ethische Positionierung im Sinne moralischer Sollensansprüche aufzeigt. Zur weiteren Debatte siehe auch Price et al. (2012). 
chend sein, nach Maßstäben der Gerechtigkeit zu suchen, die sich mit den grundlegenden Maximen aller Kulturen vereinbaren lassen (Müller 2008: 43; 2007: 111). Anstatt die kulturell-normative Fragmentierung der Weltgesellschaft nur als rhetorische Figur anzunehmen und von einem Konsens universaler Werte auszugehen, komme dem Diskurs über umstrittene Geltungsansprüche und moralische Normen - auch über Moral- und Gerechtigkeitsüberzeugungen - ein zentraler Stellenwert zu (Bjola 2009: 153-160; Deitelhoff 2009: 53; Hurrell 2005). Menschen verfügten jenseits ihrer eigenen staatlichen Grenzen über multiple Identitäten und schlössen sich im transnationalen Raum zu moralisch konstitutiven Gemeinschaften zusammen, um Solidarität und Verantwortung jenseits staatlicher Grenzen zu praktizieren (Erskine 2002: 469; O’Neill 1996: 291-302). ${ }^{37}$ Trotz partikularer Identitäten könnten Individuen unterschiedlicher Nationalitäten gemeinsame Gerechtigkeitsvorstellungen entwickeln (Erskine 2008: 179). Auf diese Weise ließen sich Konflikte und deren gewaltsame Eskalation nicht nur vermeiden, sondern auch konsensfähige Verfahren der rechtsförmigen Institutionalisierung globaler Steuerungsformen entwickeln (Appiah 2006: 4; Parekh 2002: 270-272). Sozialkonstruktivisten verweisen deshalb auf sogenannte »Diskursgemeinschaften «, die auf Grundlage gegenseitiger Anerkennung und geteilter Überzeugungen moralische Normen und politische Handlungsoptionen entwickeln (Adler 2005: 9; Price 2008; Sutch 2001).

Kritische Ansätze sehen aber auch im Ausweg der Diskursethik und Verfahrensgerechtigkeit eine Reihe von Problemen, beispielsweise das der Ungleichheit von Zugangs- und Partizipationschancen. ${ }^{38}$ Für Rainer Forst (2010: 448; 2007) wird die Analyse von Machtstrukturen und ihrer historischen Genese deshalb zur »ersten Frage der Gerechtigkeit«, die noch vor der Entwicklung von Prinzipien substanzieller Gerechtigkeit stehen müsse. ${ }^{39}$ Dahinter steht die Annahme, dass nicht die individuellen Rechte der Menschen allein Gerechtigkeitsverpflichtungen auf globaler Ebene auslösen, sondern die Grundstruktur der Globalisierung, die aus Zwang und Beherrschung bestehe. ${ }^{40}$ Einer solchen Machtanalyse nachgeschaltet ist eine gleich-

37 Erskine (2000) entwickelt hierfür auf der Grundlage von Überlegungen aus der feministischen Ethik das Konzept der »dislocated communities« und grenzt sich dadurch vom territorial begrenzten, kommunitaristischen Konzept der »communities of place« ab. Zwar bleibe der Staat für moralisch handelnde Akteure relevant, jedoch stünden sie gleichzeitig in einem »web of multiple, multifarious, overlapping and often non-territorially defined communities« (Erskine 2000: 575).

38 Kritik an der Plausibilität und Praktikabilität der Diskursethik wird auch von Vertreterinnen einer feministischen Ethik geäußert. Siehe dazu Hutchings (2010: 47, 210).

39 Owens (2007: 134) kritisiert Habermas' Diskursethik dafür, dass dieser in seiner historischen Soziologie die imperialen Entstehungsbedingungen derselben zu wenig berücksichtigt habe.

40 Der Fokus auf eine Grundstruktur von Zwang und Beherrschung, die globale Gerechtigkeitspflichten auslöst, erinnert an Galtungs Konzept der strukturellen Gewalt. Galtung selbst bezeichnet diese auch als »soziale Ungerechtigkeit« (Galtung 1971: 63). 
berechtigte Praxis der Rechtfertigung als Prinzip minimaler Gerechtigkeit, um zu gerechtfertigten transnationalen gesellschaftlichen Verhältnissen zu gelangen. Forst (2010: 452, 454) entwirft eine kritische Theorie transnationaler Gerechtigkeit, die es aus seiner Sicht ermöglicht, partikulare politische und kulturell differente Kontexte als Gerechtigkeitskontexte anzusehen und den Umgang mit Verschiedenheit darüber aufzulösen.

Solchen Mechanismen der Exklusion haben auch Feministinnen wie Iris Marion Young oder Nancy Fraser ihre Aufmerksamkeit gewidmet. ${ }^{41}$ Young identifiziert ein System struktureller Ungerechtigkeiten auf globaler Ebene und entwickelt ein »Modell sozialer Verbundenheit« (Young 2010: 353). Gerechtigkeit lässt sich darin allein auf der realpolitischen Ebene herstellen. Nur im Rahmen diskursiver transnationaler Verständigung können lokal und regional höchst unterschiedliche Erfahrungen von Ungerechtigkeit und Ungleichheit ausgetauscht und auf diese Weise in realpolitische Forderungen nach Gerechtigkeitsprinzipien in einem »glokalen Kontext« überführt werden (Hutchings 2010: 197-221; Benhabib 2002: 36; Degener/ Rosenzweig 2006: 16; Fraser 2006). Dabei bilden subjektive (lokale) Ungleichheitserfahrungen die empirische Grundlage und zugleich auch den normativen Maßstab für die Formulierung konsensualer Gerechtigkeitsvorstellungen. Damit muss stets auch ein Perspektivenwechsel aus der Sicht der »Anderen« einhergehen (Yuval-Davis 1996: 222; 2006).

Postkoloniale feministische Ansätze verweisen auf Exklusionsmechanismen und sensibilisieren für Unterschiede in den Lebenswelten zwischen Frauen aus dem globalen Süden und aus dem Westen (Spivak 1998: 819-824). Als Konsequenz betonen sie deshalb: »the subaltern cannot speak « (Spivak 1994: 104). Gleiche Zugangs- und Partizipationschancen, wie Habermas sie idealtheoretisch annimmt und wie sie auch im liberalen Feminismus vorausgesetzt werden, bleiben den »global subalterns « verwehrt. Oftmals sprächen die privilegierten »metropolitan feminists« auf internationaler Ebene für jene unterprivilegierten Frauen (Castro Varela/Dhawan 2009: 11; Dhawan 2009: 5; Castro Varela 2006). Aus der Perspektive der Verfahrensgerechtigkeit beinhalte dies, Institutionen und Verfahren zu entwickeln, in denen solche subjektiven Ungleichheitserfahrungen ausgetragen werden können, um auf diese Weise auch wahrgenommene strukturelle Ungerechtigkeiten und Machtasymmetrien zu ermitteln. Diskursethische Verfahren blieben störungsanfäl-

41 Auch wenn die ausgewählte feministische Literatur überwiegend auf der Ebene der Gesellschaftswelt argumentiert, soll sie im Rahmen des Literaturüberblicks nicht unerwähnt bleiben, weil sie interessante Einblicke in die Problematik und Begrenztheit diskursethischer Ansätze bietet. Ein guter Überblick über die verschiedenen Ansätze innerhalb der feministischen Ethik findet sich bei Hutchings (2010: 61-66). 
lig, solange kein institutioneller Rahmen fixiert worden sei, der eine gleichberechtigte Teilnahme, Repräsentativität und die Chance zur Kontestation über Unterschiede in den Gerechtigkeitsauffassungen zwischen den Konfliktparteien sicherstellt (Deitelhoff 2012: 121). ${ }^{42}$ Einblicke in die feministische Ethik fördern deshalb die notwendige Sensibilität und Bescheidenheit für das Anliegen, globale Gerechtigkeitsmaßstäbe - auch in Fragen des Einsatzes von Gewalt - zu entwickeln.

\section{Schlussbemerkungen}

Fragen internationaler Ethik und globaler Gerechtigkeit haben im Zuge von Globalisierung, interdependenter Problemstellungen und transnationaler Konflikte an Bedeutung gewonnen, dies spiegelt sich auch in den hier diskutierten normativen Ansätzen der IPT und der IB wider. Die Frage nach der Verantwortung jenseits der eigenen staatlichen Grenzen und die Reichweite der Solidarität in der Weltgemeinschaft prägen diese überwiegend kosmopolitische Literatur. Lassen sich daraus moralische Verpflichtungen von Staaten zur Hilfeleistung bei Menschenrechtsverletzungen ableiten, die notfalls auch den Einsatz militärischer Gewalt rechtfertigen? Für Kosmopolitisten ergeben sich weltbürgerliche Gerechtigkeitsverpflichtungen entweder aus Annahmen eines ethischen Individualismus oder als Erfordernis einer spezifischen Grundstruktur der Globalisierung in Form von Ausgrenzungs-, Ausbeutungs- und Herrschaftsstrukturen. Für Kommunitaristen erschließt sich Gerechtigkeit dagegen primär innerhalb partikularer Gemeinschaften auf der Grundlage gemeinsamer Identitäten, Sprache und Lebensräume. Unterschiede zwischen beiden Ansätzen lassen sich auch anhand der Frage der Reichweite sozioökonomischer und politischer Menschenrechte verdeutlichen. Während Kommunitaristen solche globalen Verpflichtungszusammenhänge nur in Fragen fundamentaler Menschenrechte sehen, identifizieren Kosmopolitisten eine moralische Verpflichtung, auch soziale Verteilungsungerechtigkeiten in Form von Armut und Unterentwicklung weltweit zu beseitigen.

In der Debatte um eine internationale Ethik des Kriegs und des Friedens ergibt sich die Problematik aus dem Spannungsfeld zwischen dem Schutz individueller Menschenrechte und der staatlichen Souveränität. Walzer verteidigt in der kommunitaristischen Lesart des »Gerechten Krieges« die Prinzipien der Nichteinmischung und der Nichtintervention mit dem Verweis auf den Zusammenhang von

42 Im Rahmen des Frankfurter Exzellenzclusters »Die Herausbildung normativer Ordnungen« ist ein Sammelband über »Transnationale Gerechtigkeit und Demokratie« erschienen (Niesen 2012). Die Debatte zur globalen Gerechtigkeit hat sich stets auch mit der Frage der demokratischen Institutionalisierung von Herrschaft befasst. 
äußerer und innerer Souveränität von Staaten, die ihre Bürger schützen müssen. Allein schwere Menschenrechtsverletzungen rechtfertigen demzufolge die externe Intervention. Die kosmopolitische Reinterpretation der Theorie des »Gerechten Krieges « rekurriert in ihrem Grundverständnis eines ethischen Individualismus auf ein breiteres Verständnis substanzieller Menschenrechte und stellt diese über die staatliche Souveränität und die Prinzipien der Nichtintervention und Nichteinmischung. Auch zeigt sich, dass die Kriterien des »Gerechten Krieges « oftmals selektiv herangezogen werden. Als Folge findet sich hier eine Tendenz der Entgrenzung von Rechtfertigungsstrategien für militärische Gewalteinsätze, die weit über die Reichweite eines basalen Menschenrechtsschutzes hinausgehen und Interventionen mit dem Ziel demokratischer Zivilisierung rechtfertigen. Krieg wird legitim, um einen gerechteren Staat im Sinne der Gewährleistung bürgerlicher Grundfreiheiten zu schaffen oder der kommenden Institutionalisierung von Weltbürgerrechten vorzugreifen. In den kosmopolitischen Konzeptionen des »Gerechten Krieges « tritt anstelle des rechtlichen das moralische Argumentieren: Gefragt wird nicht mehr, ob ein Krieg legal ist, sondern ob er sich moralisch vertreten lässt. Somit gibt die Lehre vom »Gerechten Krieg« das große Projekt der Aufklärung - die Herrschaft eines durch praktische Vernunft gebändigten Rechts - zugunsten von Gerechtigkeitsabwägungen auf und lässt Freiheit als Willkürfreiheit erscheinen (L. Brock 2011; Koskenniemi 2009). Einmal mehr verdeutlicht sich in dieser Argumentation das Problem der kulturellen Unschärferelation kosmopolitischer Ansätze. Das auf Selbstreflexion basierende Urteil, das den Universalisierungsanspruch begründet, ist Ergebnis rationaler Vernunft, die sich als Sozialisationsergebnis jedoch in einem distinkten historisch-kulturellen, westlichen Kontext entwickelt hat (Müller 2011 b).

Kosmopolitische Überlegungen des »Gerechten Krieges« befinden sich auf dünnem Eis, da die Bemühungen um Universalisierung von Menschenrechten im Partikularen enden, indem sie zur Instrumentalisierung solcher universeller Normen durch mächtige Staaten beitragen. Der Hinweis liberaler Internationalisten auf die besondere Verantwortung liberaler Demokratie für die notfalls auch gewaltsame Zivilisierung von Unrechtsregimen verdeutlicht die Idiosynkrasien moralisch gerechtfertigter Weltordnungspolitik und bestätigt die Sorge nicht-westlicher Staaten vor westlich dominierten, universalistischen Herrschaftsansprüchen (Jahn 2012: 54). Auf der Grundlage liberaler Argumentation wird hier ein Zivilisationsstandard konstruiert, der Widerstand hervorruft. Derartige Überlegungen konterkarieren neuere Ansätze der Gerechtigkeitsforschung, die darauf abzielen, der vielerorts artikulierten Sorge entgegenzuwirken, die kulturell-normative Fragmentierung der Weltgesellschaft stelle ein Bedrohungsszenario dar. Aus einer solchen Perspektive 
kommt deshalb dem Diskurs über umstrittene Geltungsansprüche von Normen, also auch Moral- und Gerechtigkeitsansprüchen, eine zentrale Bedeutung zu. Gerechtigkeit ist ein Zustand, in dem Akteure das erhalten, was ihnen zusteht (Gosepath 2008). In Verhandlungen wird Gerechtigkeit dann zunächst zum Sprechakt mit hohem Konfliktpotenzial. Mittels deliberativer Institutionen oder Verfahrensgerechtigkeit sollen Akteure ihre Differenzen über substanzielle Fragen der Gerechtigkeit austragen. Doch bleibt zu vermuten, dass Problemlösungen für kollektiven Regelungsbedarf so lange umstritten bleiben und schwierig umzusetzen sind, wie zumindest minimale Gerechtigkeitsvorstellungen der beteiligten Akteure unbefriedigt bleiben (Daase 2011; Albin 2001; Müller 2011 a; 2011 b). Dabei scheinen gerade fundamentale Normen, wie das Verständnis der Reichweite von Menschenrechten oder die Interpretation von Souveränität, zwischen den Staaten besonders kontrovers zu sein (Wiener 2011: 109). Sie belasten Diskursgemeinschaften in der Entwicklung konsensualer Gerechtigkeitsvorstellungen und somit auch die Entstehung rechtsförmiger globaler Regulierungen.

Zusammengefasst bedeutet dies, dass die Frage nach der Verantwortung jenseits staatlicher Grenzen im Hinblick auf die Reichweite der Verpflichtungen und die Frage des Gewalteinsatzes die Notwendigkeit einer stärker empirisch ausgerichteten Gerechtigkeitsforschung unterstreicht, die sich am Ist-Zustand orientiert, um unter anderem zu ermitteln, ob und inwieweit sich in der Weltgesellschaft bzw. in der Staatenwelt universelle Gerechtigkeitsüberzeugungen abzeichnen und wo ihre Differenzen und Friktionen sowie weiterer Aushandlungsbedarf bestehen (Liebig/ Lengfeld 2002). Erste empirische Untersuchungen in staatlichen Verhandlungen zu globalen Regulierungen unterstreichen die Signifikanz der Umstrittenheit von Gerechtigkeitsvorstellungen, aber auch die Chancen auf Vereinbarung globaler Normen und Regulierungen (Müller/Wunderlich 2013; Albin 2001). Für die Theoriebildung zur globalen Gerechtigkeit wäre damit Amartya Sen $(2010 ; 2002)$ zu folgen, der idealtypische und normativ gehaltvolle Konzeptionen globaler Gerechtigkeit ablehnt und fordert, am realpolitischen Ist-Zustand anzusetzen, um die auf diese Weise auf globaler Ebene kollektiv ermittelten Strukturen der Ungerechtigkeit zumindest ein Stück weit zu revidieren.

\section{Literatur}

Adler, Emanuel 2005: Communitarian International Relations. The Epistemic Foundations of International Relations, New York, NY.

Albin, Cecilia 2001: Justice and Fairness in International Negotiation, Cambridge. Appiah, Kwame A. 2006: Cosmopolitanism. Ethics in a World of Strangers, London. 
Ayoob, Mohammed 2004: Third World Perspectives on Humanitarian Intervention and International Administration, in: Global Governance 10: 1, 99-118.

Bajpai, Kanti 2003: Indian Conception of Order and Justice: Nehruvian, Gandhian, Hindutva, and Neo-Liberal, in: Foot, Rosemary/Gaddis, John Lewis/Hurrell, Andrew (Hrsg.): Order and Justice in International Relations, Oxford, 236-261. Barnett, Michael 2005: Humanitarianism Transformed, in: Perspectives on Politics 3: 4, 723-740.

Beardsworth, Richard 2011: Cosmopolitanism and International Relations Theory, Cambridge.

Beitz, Charles 1979: Political Theory and International Relations, Princeton, NJ.

Bellamy, Alex J. 2005: Responsibility to Protect or Trojan Horse? The Crisis in

Darfur and Humanitarian Intervention after Iraq, in: Ethics \& International Affairs 19: 2, 31-53.

Bellamy, Alex J. 2006: Just Wars. From Cicero to Iraq, London.

Bellamy, Alex J. 2009: Responsibility to Protect. The Global Effort to End Mass Atrocities, Cambridge.

Benhabib, Seyla 2002: The Claims of Culture: Equality and Diversity in the Global Era, Princeton, NJ.

Bishai, Linda S. 2004: Liberal Empire, in: Journal of International Relations and Development 7: 1, 48-72.

Bjola, Corneliu 2009: Legitimising the Use of Force in International Politics: Kosovo, Iraq \& the Ethics of Intervention, London.

Brock, Gillian 2009: Global Justice. A Cosmopolitan Account, Oxford.

Brock, Gillian/Brighouse, Harry (Hrsg.) 2005: The Political Philosophy of Cosmopolitanism, Cambridge.

Brock, Lothar 2002: »Staatenrecht« und »Menschenrecht«. Schwierigkeiten der Annäherung an eine weltbürgerliche Ordnung, in: Bohman, James/Lutz-Bachmann, Mathias (Hrsg.): Weltstaat oder Staatenwelt?, Frankfurt a. M., 201-225.

Brock, Lothar 2007: Universalismus, politische Heterogenität und ungleiche Entwicklung. Internationale Kontexte der Gewaltanwendung von Demokratien gegenüber Nichtdemokratien, in: Geis, Anna/Müller, Harald/Wagner, Wolfgang (Hrsg.): Schattenseiten des Demokratischen Friedens. Zur Kritik einer Theorie liberaler Außen- und Sicherheitspolitik, Frankfurt a. M., 45-68.

Brock, Lothar 2011: Von der liberalen Universalpoesie zu reflexiver Friedenspolitik! Die Demokratie als Medium einer brisanten Vermittlung zwischen Frieden und Gerechtigkeit, in: Baumgart-Ochse, Claudia/Schörnig, Niklas/Wisotzki, Simone/Wolff, Jonas (Hrsg.): Auf dem Weg zu Just Peace Governance. Beiträge zum Auftakt des neuen Forschungsprogramms der HSFK, Baden-Baden, 47-70. 
Broszies, Christoph/Hahn, Henning 2010: Die Kosmopolitismus-PartikularismusDebatte im Kontext, in: dies. (Hrsg.): Globale Gerechtigkeit. Schlüsseltexte zur Debatte zwischen Partikularismus und Kosmopolitismus, Frankfurt a. M., 9-52. Brown, Chris 1992: International Relations Theory. New Normative Approaches, New York, NY.

Brown, Chris 2002: Sovereignty, Rights and Justice. International Political Theory Today, Cambridge.

Buchanan, Allen 2003: Reforming the International Law of Humanitarian Intervention, in: Holzgrefe, J. L./Keohane, Robert O. (Hrsg.): Humanitarian Intervention. Ethical, Legal, and Political Dilemmas, Cambridge, 130-174.

Buchanan, Allen/Keohane, Robert O. 2004: The Preventive Use of Force: A Cosmopolitan Institutional Proposal, in: Ethics \& International Affairs 18: 1, 1-22. Buchanan, Allen/Keohane, Robert O. 2005: Justifying Preventive Force: Reply to Steven Lee, in: Ethics \& International Affairs 19: 3, 109-112.

Bull, Hedley 1977: The Anarchical Society, New York, NY.

Burke, Anthony 2004: Just War or Ethical Peace? Moral Discourse of Strategic Violence after 9/11, in: International Affairs 80: 2, 329-353.

Burke, Anthony 2005: Against the New Internationalism, in: Ethics \& International Affairs 19: 2, 73-89.

Buzan, Barry 2004: From International to World Society? English School Theory and the Social Structure of Globalization, Cambridge.

Campbell, David/Shapiro, Michael J. 1999: Introduction: From Ethical Theory to the Ethical Relation, in: dies. (Hrsg.): Moral spaces. Rethinking Ethics and World Politics, Minneapolis, MN, VII-XX.

Caney, Simon 2005: Justice beyond Borders: A Global Political Theory, Oxford.

Caney, Simon 2010: Cosmopolitanism, in: Bell, Duncan (Hrsg.): Ethics and World Politics, Oxford, 146-163.

Castro Varela, Maria do Mar 2006: Postkoloniale feministische Theorie und soziale Gerechtigkeit, in: Degener, Ursula/Rosenzweig, Beate (Hrsg.): Die Neuverhandlung sozialer Gerechtigkeit. Feministische Analysen und Perspektiven, Wiesbaden, 97-114.

Castro Varela, Maria do Mar/Dhawan, Nikita 2009: Feministische Postkoloniale Theorie: Gender und (De-)Kolonisierungsprozesse, in: Femina Politica 2/2009, 2-9.

Cochran, Molly 1999: Normative Theory in International Relations. A Pragmatic Approach, Cambridge.

Daase, Christopher 2011: »Just Peace Governance« - Auf dem Weg zu einem neuen Forschungsprogramm, in: Baumgart-Ochse, Claudia/Schörnig, Niklas/ 
Wisotzki, Simone/Wolff, Jonas (Hrsg.): Auf dem Weg zu Just Peace Governance. Beiträge zum Auftakt des neuen Forschungsprogramms der HSFK, Baden-Baden, 17-26.

Degener, Ursula/Rosenzweig, Beate 2006: Einleitung: Die Neuverhandlung sozialer Gerechtigkeit - eine kritische Bestandsaufnahme, in: dies. (Hrsg.): Die Neuverhandlung sozialer Gerechtigkeit. Feministische Analysen und Perspektiven, Wiesbaden, 11-33.

Deitelhoff, Nicole 2006: Überzeugung in der Politik. Grundzüge einer Diskurstheorie, Frankfurt a. M.

Deitelhoff, Nicole 2007: Demokratische Bindungsängste? Die ambivalente Beziehung zwischen nationaler Demokratie und internationalem Recht, in: Geis, Anna/Müller, Harald/Wagner, Wolfgang (Hrsg.): Schattenseiten des Demokratischen Friedens. Zur Kritik einer Theorie liberaler Außen- und Sicherheitspolitik, Frankfurt a. M., 147-172.

Deitelhoff, Nicole 2009: Was vom Tage übrig blieb. Inseln der Überzeugung im vermachteten Alltagsgeschäft internationalen Regierens, in: Niesen, Peter/Herborth, Benjamin (Hrsg.): Anarchie der kommunikativen Freiheit. Jürgen Habermas und die Theorie der internationalen Politik, Frankfurt a. M., 26-56.

Deitelhoff, Nicole 2012: (Is) Fair Enough? - Legitimation internationalen Regierens durch deliberative Verfahren, in: Niesen 2012, 103-130.

Desch, Michael C. 2007: America's Liberal Illiberalism: The Ideological Origins of Overreaction in U.S. Foreign Policy, in: International Security 32: 3, 7-43.

Dhawan, Nikita 2009: Zwischen Empire und Empower: Dekolonisierung und Demokratisierung, in: Femina Politica 2/2009, 52-63.

Duffield, Mark 2001: Global Governance and the New Wars. The Merging of Development and Security, New York, NY.

Dunne, Tim 1998: Inventing International Society: A History of the English School, London.

Dunne, Tim/Wheeler, Nicolas J. 2004: »We the Peoples«: Contending Discourses of Security in Human Rights Theory and Practice, in: International Relations 18: 1, 9-23.

Elshtain, Jean Bethke 2003: International Justice as Equal Regard and the Use of Force, in: Ethics \& International Affairs 17: 2, 63-75.

Erskine, Toni 2000: Embedded Cosmopolitanism and the Case of War: Restraint, Discrimination and Overlapping Communities, in: Global Society 14: 4, 569-590. 
Erskine, Toni 2002: 〉Citizen of Nowhere or \the Point where Circles Intersect $<$ ? Impartialist and Embedded Cosmopolitanisms, in: Review of International Studies 28: 3, 457-478.

Erskine, Toni 2008: Embedded Cosmopolitanism: Duties to Strangers and Enemies in a World of 〉Dislocated Communities`, Oxford.

Evans, Gareth 2009: The Responsibility to Protect. Ending Mass Atrocity Crimes Once and For All, Washington, D.C.

Evans, Gareth/Sahnoun, Mohamed 2002: The Responsibility to Protect, in: Foreign Affairs 81: 6, 99-110.

Fabre, Cécile 2008: Cosmopolitanism, Just War Theory and Legitimate Authority, in: International Affairs 84: 5, 963-976.

Falk, Richard 2005: Legality and Legitimacy: The Quest for Principled Flexibility and Restraint, in: Review of International Studies 31: 3, 33-50.

Farer, Tom J. 2003: Humanitarian Intervention before and after 9/11: Legality and Legitimacy, in: Holzgrefe, J.L./Keohane, Robert O. (Hrsg.): Humanitarian Intervention. Ethical, Legal, and Political Dilemmas, Cambridge, 53-90.

Feinstein, Lee/Slaughter, Anne-Marie 2004: A Duty to Prevent, in: Foreign Affairs 83: 1, 136-150.

Forst, Rainer 2007: Dialektik der Moral. Grundlagen einer Diskurstheorie transnationaler Gerechtigkeit, in: Niesen, Peter/Herborth, Benjamin (Hrsg.): Anarchie der kommunikativen Freiheit. Jürgen Habermas und die Theorie der internationalen Politik, Frankfurt a. M., 254-268.

Forst, Rainer 2010: Zu einer kritischen Theorie transnationaler Gerechtigkeit, in: Broszies, Christoph/Hahn, Henning (Hrsg.): Globale Gerechtigkeit, Frankfurt a. M., 439-464.

Fraser, Nancy 2006: Mapping the Feminist Imagination: From Redistribution to Recognition to Representation, in: Degener, Ursula/Rosenzweig, Beate (Hrsg.): Die Neuverhandlung sozialer Gerechtigkeit. Feministische Analysen und Perspektiven, Wiesbaden, 37-52.

Fraser, Nancy 2008: Scales of Justice. Reimagining Political Space in a Globalizing World, Cambridge.

Frost, Mervyn 2009: Global Ethics. Anarchy, Freedom and International Relations, New York, NY.

Galtung, Johan 1971: Gewalt, Frieden und Friedensforschung, in: Senghaas, Dieter (Hrsg.): Kritische Friedensforschung, Frankfurt a. M., 55-104.

Geis, Anna/Müller, Harald/Wagner, Wolfgang 2007 a: Einleitung. Das Kantsche Friedensprogramm und seine Schattenseiten, in: dies. (Hrsg.): Schattenseiten des 
Demokratischen Friedens. Zur Kritik einer Theorie liberaler Außen- und Sicherheitspolitik, Frankfurt a. M., 11-42.

Geis, Anna/Brock, Lothar/Müller, Harald 2007 b: Demokratische Kriege als Antinomien des Demokratischen Friedens: Eine komplementäre Forschungsagenda, in: Geis, Anna/Müller, Harald/Wagner, Wolfgang (Hrsg.): Schattenseiten des Demokratischen Friedens. Zur Kritik einer Theorie liberaler Außen- und Sicherheitspolitik, Frankfurt a. M., 69-92.

Glanville, Luke 2010: The Antecedents of >Sovereignty as Responsibility<, in: European Journal of International Relations 17: 2, 233-255.

Goldstein, Jürgen 2012: Perspektiven des politischen Denkens. Sechs Portraits, Weilerswist.

Gong, Gerrit W. 1984: The Standard of »Civilization« in International Society, Oxford.

Gosepath, Stefan 2008: Gleiche Gerechtigkeit. Grundlagen eines liberalen Egalitarismus, Frankfurt a. M.

Habermas, Jürgen 1999 a: Theorie des kommunikativen Handelns. Handlungsrationalität und gesellschaftliche Rationalisierung (Band 1 und 2), Frankfurt a. M.

Habermas, Jürgen 1999 b: Bestialität und Humanität. Ein Krieg an der Grenze zwischen Recht und Moral, in: Die Zeit, 29.4.1999, http://pdf.zeit.de/1999/18/1999 18.krieg_.xml.pdf; 16.3.2013.

Habermas, Jürgen 2004: Der gespaltene Westen, Frankfurt a. M.

Hasenclever, Andreas 2000: Die Macht der Moral in der internationalen Politik. Militärische Interventionen westlicher Staaten in Somalia, Ruanda und BosnienHerzegowina, Frankfurt a M.

Haspel, Michael 2002: Friedensethik und Humanitäre Intervention. Der KosovoKrieg als Herausforderung evangelischer Friedensethik, Neukirchen-Vluyn.

Hayden, Patrick 2005: Security Beyond the State: Cosmopolitanism, Peace, and the Role of Just War Theory, in: Evans, Mark (Hrsg.): Just War Theory. A Reappraisal, Edinburgh, 157-176.

Hehir, Aidan 2010: Humanitarian Intervention. An Introduction, Basingstoke.

Hobson, John M. 2008: The Eastern Origins of Western Civilisation, Cambridge.

Hoffmann, Matthew 2009: Is Constructivist Ethics an Oxymoron?, in: International Studies Review 11: 2, 231-252.

Hudson, Kimberly A. 2009: Justice, Intervention, and Force in International Relations: Reassessing Just War Theory in the 21st Century, New York.

Hurrell, Andrew 2003: Order and Justice in International Relations: What is at Stake?, in: Foot, Rosemary/Gaddis, John Lewis/Hurrell, Andrew (Hrsg.): Order and Justice in International Relations, Oxford, 24-48. 
Hurrell, Andrew 2005: Legitimacy and the Use of Force: Can the Circle be Squared?, in: Review of International Studies 31: Supplement S1, 15-32.

Hutchings, Kimberly 1999: International Political Theory: Rethinking Ethics in a Global Era, London.

Hutchings, Kimberly 2010: Global Ethics. An Introduction, Cambridge.

Imbusch, Peter (Hrsg.) 2007: Gerechtigkeit - Demokratie - Frieden. Eindämmung oder Eskalation von Gewalt?, Baden-Baden.

Jaberg, Sabine/Schlotter, Peter (Hrsg.) 2005: Imperiale Weltordnung - Trend des 21. Jahrhunderts?, Baden-Baden.

Jabri, Vivienne 2007 a: War and the Transformation of Global Politics, New York, NY.

Jabri, Vivienne 2007 b: Solidarity and Spheres of Culture: The Cosmopolitan and the Postcolonial, in: Review of International Studies 33: 4, 715-728.

Jackson, Robert H. 1990: Quasi-states: Sovereigny, International Relations and the Third World, Cambridge.

Jackson, Robert H. 2000: The Global Covenant: Human Conduct in a World of States, Oxford.

Jahn, Beate 2012: Humanitarian Intervention: What's in a Name?, in: International Politics 49: 1, 36-58.

Koskenniemi, Martti 2009. The Politics of International Law - 20 Years Later, in: European Journal of International Law 20: 1, 7-19.

Lang, Anthony F. 2010: Humanitarian Intervention; in: Bell, Duncan (Hrsg.): Ethics and World Politics, Oxford, 324-341.

Liebig, Stefan/Lengfeld, Holger (Hrsg.) 2002: Interdisziplinäre Gerechtigkeitsforschung. Zur Verknüpfung empirischer und normativer Perspektiven, Hamburg. Linklater, Andrew 2002: Cosmopolitan Political Communities in International Relations, in: International Relations 16: 1, 135-150.

Linklater, Andrew 2006: The Harm Principle and Global Ethics, in: Global Society 20: 3, 329-343.

Lu, Catherine 2000: The One and Many Faces of Cosmopolitanism, in: Journal of Political Philosophy 8: 2, 244-267.

Lynch, Mark 2000: The Dialogue of Civilizations and International Public Spheres, in: Millennium 29: 2, 307-330.

MacFarlane, Neil S./Thielking, Carolin J./Weiss, Thomas G. 2004: The Responsibility to Protect: Is Anyone Interested in Humanitarian Intervention?, in: Third World Quarterley 25: 5, 977-992. 
Mayer, Peter 1999: War der Krieg der NATO gegen Jugoslawien moralisch gerechtfertigt? Die Operation »Allied Force« im Lichte der Lehre vom gerechten Krieg, in: Zeitschrift für Internationale Beziehungen 6: 2, 287-321.

Merkel, Reinhard 2004: Können Menschenrechtsverletzungen militärische Interventionen rechtfertigen? Rechtsethische Grundlagen und Grenzen der »Humanitären Intervention« am Beispiel des Kosovo-Krieges, in: Meggle, Georg (Hrsg.): Humanitäre Interventionsethik. Was lehrt uns der Kosovo-Krieg?, Paderborn, 107-132.

Miller, David 1999: On Nationality, Oxford.

Miller, David 2005: Global Egalitarianism, in: The Journal of Ethics 9: 1/2, 55-79.

Miller, David 2007: National Responsibility and Global Justice, Oxford.

Mills, Kurt 2005: Neo-Humanitarianism: The Role of International Humanitarian

Norms and Organizations in Contemporary Conflict, in: Global Governance 11:

2, 161-183.

Moellendorf, Darrel 2002: Cosmopolitan Justice, Oxford.

Moses, Jeremy 2010: Liberal Internationalist Discourse and the Use of Force: Blair,

Bush and Beyond, in: International Politics 47:1, 26-51.

Müller, Harald 2007: Internationale Verhandlungen, Argumente und Verständigungshandeln. Verteidigung, Befunde, Warnungen, in: Niesen, Peter/Herborth, Benjamin (Hrsg.): Anarchie der kommunikativen Freiheit. Jürgen Habermas und die Theorie der internationalen Politik, Frankfurt a. M., 199-223.

Müller, Harald 2008: Wie kann eine neue Weltordnung aussehen? Wege in eine nachhaltige Politik, Frankfurt a. M.

Müller, Harald 2011 a: Justice in International Diplomacy (PRIF Working Paper No. 8), in: http://www.hsfk.de/PRIF-Working-Papers.891.0.html; 8.4.2013.

Müller, Harald 2011 b: Demokratie, Gerechtigkeit und Frieden: Die guten Dinge gehen nicht immer zusammen, in: Baumgart-Ochse, Claudia/Schörnig, Niklas/ Wisotzki, Simone/Wolff, Jonas (Hrsg.): Auf dem Weg zu Just Peace Governance. Beiträge zum Auftakt des neuen Forschungsprogramms der HSFK, Baden-Baden, 277-310.

Müller, Harald/Wunderlich, Carmen 2013: Norm Dynamics in Multilateral Arms Control: Interests, Conflicts, and Justice, Athens, GA.

Mutua, Makau 2001: Savages, Victims, and Saviors: The Metaphor of Human Rights, in: Harvard International Law Journal 42: 1, 201-245.

Nagel, Thomas 2005: The Problem of Global Justice, in: Philosophy and Public Affairs 33: 2, 115-147.

Nardin, Terry 1983: Law, Morality and the Relations of States, Princeton, NJ. 
Nardin, Terry 2002: The Moral Basis of Humanitarian Intervention, in: Ethics \& International Affairs 16: 1, 57-70.

Niesen, Peter (Hrsg.) 2012: Transnationale Gerechtigkeit und Demokratie, Frankfurt a. M.

Nussbaum, Martha C. 2006: Frontiers of Justice. Disability, Nationality, Species Membership, Cambridge, MA.

O'Neill, Onora 1996: Gerechtigkeit, Geschlechterdifferenz und internationale Grenzen, in: Nagl-Docekal, Herta/Pauer-Studer, Herlinde (Hrsg.): Politische Theorie: Differenz und Lebensqualität, Frankfurt a. M., 417-450.

Owens, Patricia 2007: Between War and Politics. International Relations and the Thought of Hannah Arendt, Oxford.

Parekh, Bhikhu 2002: Rethinking Multiculturalism: Cultural Diversity and Political Theory, Cambridge, MA.

Pogge, Thomas 1992: Cosmopolitanism and Sovereignty, in: Ethics 103: 1, 48-75. Pogge, Thomas 2002 a: World Poverty and Human Rights: Cosmopolitan Responsibilities and Reforms, Cambridge, MA.

Pogge, Thomas 2002 b: Human Rights and Human Responsibilities, in: Greiff, Pablo de/Cronin, Ciaran (Hrsg.): Global Justice and Transnational Politics. Essays on the Moral and Political Challenges of Globalization, Cambridge, MA, 151-195.

Pogge, Thomas 2007: Severe Poverty as Human Rights Violation, in: ders. (Hrsg.): Freedom from Poverty as Human Right. Who Owes What to the Very Poor?, Oxford, 11-54.

Price, Richard 2008: Moral Limit and Possibility in World Politics, Cambridge.

Price, Richard/Snyder, Jack/Vinjamuri, Leslie/Erskine, Toni/Rengger, Nicholas 2012: Special Forum on Moral Limit and Possibility in World Politics, in: International Theory 4: 3, 430-434.

Purdy, Jedediah 2005: The New Liberal Imperialism: Assessing the Arguments, in: Barry, Christian/Pogge, Thomas (Hrsg.): Global Institutions and Responsibilities. Achieving Global Justice, Oxford, 323-338.

Rawls, John 1972: A Theory of Justice, London.

Rawls, John 1999: The Law of Peoples, Cambridge, MA.

Rawls, John 2001: Gerechtigkeit als Fairness, Frankfurt a. M.

Reus-Smit, Christian 2005: Liberal Hierarchy and the Licence to Use Porce, in:

Review of International Studies 31: Supplement S1, 71-92. 
Risse, Thomas 2007: Global Governance und kommunikatives Handeln, in: Niesen, Peter/Herborth, Benjamin (Hrsg.): Anarchie der kommunikativen Freiheit. Jürgen Habermas und die Theorie der internationalen Politik, Frankfurt a. M., 57-86.

Roennfeldt, Carsten F. 1999: Beyond a Pluralist Conception of International Society? A Case Study on the International Response, in: Cooperation and Conflict 34: 2, 141-168.

Schmidt, Hajo 2004: Wie weiter? Rechtsethische Erwägungen anlässlich des Kosovo-Krieges, in: Meggle, Georg (Hrsg.): Humanitäre Interventionsethik. Was lehrt uns der Kosovo-Krieg?, Paderborn, 241-265.

Sen, Amartya 2002: Justice across Borders, in: Greiff, Pablo de/Cronin, Ciaran (Hrsg.): Global Justice and Transnational Politics. Essays on the Moral and Political Challenges of Globalization, Cambridge, MA, 37-51.

Sen, Amartya 2010: The Idea of Justice, London.

Senghaas, Dieter 1998: Zivilisierung wider Willen. Der Konflikt der Kulturen mit sich selbst, Frankfurt a. M.

Shapcott, Richard 2010: International Ethics. A Critical Introduction, Cambridge. Smith, Michael Joseph 1992: Liberalism and International Reform, in: Nardin, Terry/Mapel, David R. (Hrsg.): Traditions of International Ethics, Cambridge, 201-224.

Spivak, Gayatri Chakravorty 1994: Can the Subaltern Speak, in: Williams, Patrick/ Chrisman, Laura (Hrsg.): Colonial Discource and Post-Colonial Theory, New York, NY, 66-111.

Spivak, Gayatri Chakravorty 1998: Gender and International Studies, in: Millennium 27: 4, 809-831.

Stahn, Carsten 2007: Responsibility to Protect: Political Rhetoric or Emerging legal Norm?, in: The American Journal of International Law 101: 1, 99-120.

Sutch, Peter 2001: Ethics, Justice and International Relations, New York, NY.

Tan, Kok-Chor 2004: Justice Beyond Borders: Cosmopolitanism, Nationalism, and Patriotism, Cambridge.

Tesón, Fernando R. 2003: The Liberal Case for Humanitarian Intervention, in: Holzgrefe, J.L./Keohane, Robert O. (Hrsg.): Humanitarian Intervention. Ethical, Legal, and Political Dilemmas, Cambridge, 93-129.

Tesón, Fernando R. 2005: Ending Tyranny in Iraq, in: Ethics \& International Affairs 19: 1, 1-20.

Vincent, R.J. 1986: Human Rights and International Relations. Issues and Responses, Cambridge. 
Walzer, Michael 1977: Just and Unjust Wars. A Moral Argument with Historical Illustrations, New York, NY.

Walzer, Michael 1994: Thick and Thin: Moral Argument at Home and Abroad, Notre Dame, IN.

Walzer, Michael 2002: The Triumph of Just War Theory (and the Dangers of Success), in: Social Research 69: 4, 925-944.

Walzer, Michael 2003: Erklärte Kriege - Kriegserklärungen, Hamburg.

Walzer, Michael 2006: Sphären der Gerechtigkeit. Ein Plädoyer für Pluralität und Gleichheit, Frankfurt a. M.

Welsh, Jennifer M. 2010: Die internationale Gemeinschaft und die »Veranwortung zum Schutz«, in: Gerber, Doris/Zanetti, Véronique (Hrsg.): Kollektive Verantwortung und Internationale Beziehungen, Frankfurt a. M., 272-294.

Wheeler, Nicholas J. 1992: Pluralist or Solidarist Conceptions of International Society, in: Millennium 21: 3, 463-487.

Wheeler, Nicholas J. 2000: Saving Strangers. Humanitarian Intervention in International Society, Oxford.

Wiener, Antje 2011: Cultural Validation: Examining the Familiarity Deficit of Global Governance, in: Bjola, Corneliu/Kornprobst, Markus (Hrsg.): Arguing Global Governance, London, 103-116.

Young, Iris Marion 2010: Verantwortung und globale Gerechtigkeit. Ein Modell sozialer Verbundenheit, in: Broszies, Christoph/Hahn, Henning (Hrsg.): Globale Gerechtigkeit, Frankfurt a. M., 329-369.

Yuval-Davis, Nira 1996: Frauen und transversale Politik, in: Fuchs, Brigitte/Habinger, Gabriele (Hrsg.): Rassismus und Feminismus. Differenzen, Machtverhältnisse und Solidarität, Frankfurt a. M., 217-223.

Yuval-Davis, Nira 2006: Human/Women Rights and Feminist Transversal Politics, in: Marx Ferree, Myra/Tripp, Aili Mari (Hrsg.): Global Feminism. Transnational Women's Activism, Organizing, and Human Rights, New York, NY, 275-295.

\section{Die Autorin}

Dr. Simone Wisotzki ist wissenschaftliche Mitarbeiterin der Hessischen Stiftung Friedens- und Konfliktforschung (HSFK) in Frankfurt am Main.

E-Mail: wisotzki@hsfk.de 